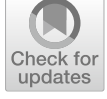

Cite as

Nano-Micro Lett.

(2021) 13:77

Received: 25 November 2020

Accepted: 12 January 2021

Published online: 19 February 2021

(C) The Author(s) 2021

\section{N-Doped Graphene-Decorated NiCo Alloy Coupled with Mesoporous NiCoMoO Nano-sheet Heterojunction for Enhanced Water Electrolysis Activity at High Current Density}

\author{
Guangfu Qian ${ }^{1}$, Jinli Chen ${ }^{1}$, Tianqi Yu$^{1}$, Lin Luo ${ }^{1}$, Shibin Yin ${ }^{1} 凶$
}

\title{
HIGHLIGHTS
}

- $\mathrm{N}$-doped graphene-coated structure and mesoporous nano-sheet can efficiently boost active sites and stability for hydrogen and oxygen evolution reaction.

- NiCo@C-NiCoMoO/NF exhibits low overpotentials for HER $(266 \mathrm{mV})$ and OER $(390 \mathrm{mV})$ at $\pm 1000 \mathrm{~mA} \mathrm{~cm}$.

- For water electrolysis, it can hold at $1000 \mathrm{~mA} \mathrm{~cm}{ }^{-2}$ for $43 \mathrm{~h}$ in $6.0 \mathrm{M} \mathrm{KOH}+60{ }^{\circ} \mathrm{C}$ condition.

\begin{abstract}
Developing highly effective and stable non-noble metalbased bifunctional catalyst working at high current density is an urgent issue for water electrolysis (WE). Herein, we prepare the N-doped graphene-decorated NiCo alloy coupled with mesoporous $\mathrm{NiCoMoO}$ nano-sheet grown on 3D nickel foam (NiCo@C-NiCoMoO/NF) for water splitting. NiCo@C-NiCoMoO/NF exhibits outstanding activity with low overpotentials for hydrogen and oxygen evolution reaction (HER: 39/266 mV; OER: 260/390 mV) at \pm 10 and $\pm 1000 \mathrm{~mA} \mathrm{~cm}^{-2}$. More importantly, in $6.0 \mathrm{M} \mathrm{KOH}$ solution at $60{ }^{\circ} \mathrm{C}$ for WE, it only requires $1.90 \mathrm{~V}$ to reach $1000 \mathrm{~mA} \mathrm{~cm}^{-2}$ and shows excellent stability for $43 \mathrm{~h}$, exhibiting the potential for actual application. The good performance can be assigned to $\mathrm{N}$-doped graphene-decorated $\mathrm{NiCo}$ alloy and mesoporous NiCoMoO nano-sheet, which not only increase the intrinsic activity and expose abundant catalytic activity sites, but
\end{abstract}

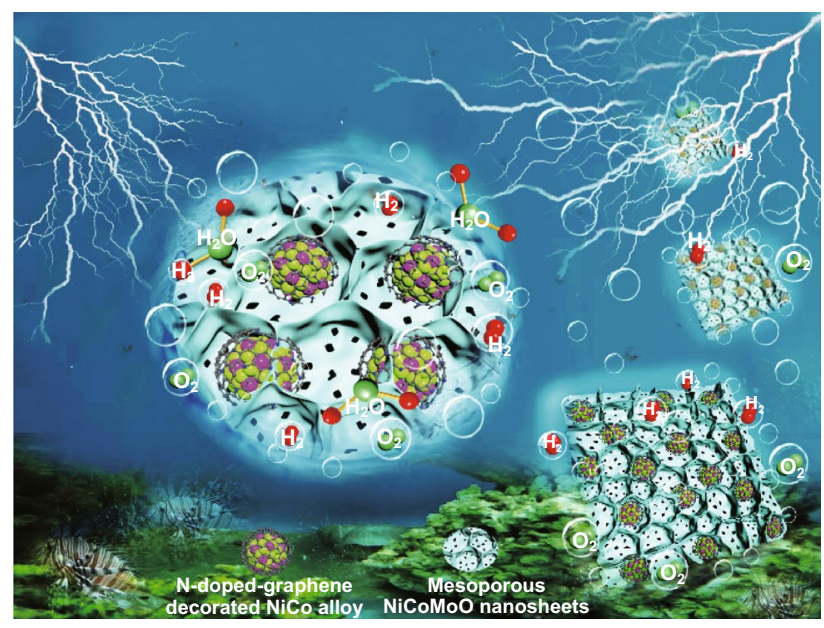
also enhance its chemical and mechanical stability. This work thus could provide a promising material for industrial hydrogen production. KEYWORDS N-doped graphene-decorated NiCo alloy; Catalyst; Mesoporous nano-sheet; Water electrolysis; High current density

${ }^{1}$ College of Chemistry and Chemical Engineering, MOE Key Laboratory of New Processing Technology for Nonferrous Metals and Materials, State Key Laboratory of Processing for Non-Ferrous Metal and Featured Materials, Guangxi University, 100 Daxue Road, Nanning 530004, P. R. China 


\section{Introduction}

Water electrolysis (WE) can convert renewable sources (i.e., solar, wind) into $\mathrm{H}_{2}$ with clean and high energy density, but the sluggish kinetics of hydrogen and oxygen evolution reaction (HER and OER) at cathode and anode will hinder its efficiency [1-4]. Although Pt-/Ir-/Ru-based materials are the best choice to accelerate these two half-reactions, the large-scale hydrogen production is still limited by its shortage and high price [5-7]. Therefore, developing highly efficient non-precious metal materials to replace the noble metals for reducing cost and improving the performance of WE are necessary $[8,9]$.

Recently, 3D transition metal-based (TMB) catalysts are regarded as prospective alternative to noble metals, due to their abundance and low cost [10-14]. However, the 3D TMB catalysts are unstable under strong alkaline conditions. To address this problem, some researchers reported a novel strategy to construct the 3D TMB catalysts with $\mathrm{N}$-doped graphene-encapsulated to improve the stability and catalytic activity [15]. Deng et al. prepared the ultrathin graphene layer encapsulating FeNi alloy and efficiently optimizing its surface electronic structure [16], and it obtains a low overpotential $(280 \mathrm{mV})$ at $10 \mathrm{~mA} \mathrm{~cm}{ }^{-2}$ for OER and can keep for $24 \mathrm{~h}$. Mu et al. fabricated a $\mathrm{Mo}_{2} \mathrm{C} @ \mathrm{C}$ nanoball with hollow porous, which displayed low overpotentials for HER in $1.0 \mathrm{M}$ $\mathrm{KOH}(115 \mathrm{mV})$ and $0.5 \mathrm{M} \mathrm{H}_{2} \mathrm{SO}_{4}(129 \mathrm{mV})$ solution at $-10 \mathrm{~mA} \mathrm{~cm}^{-2}$ [17]. Furthermore, other investigators also use the N-doped carbon-encapsulated 3D TMB catalysts, which can optimize the distribution of electrons on the metal surface and prevent metal dissolution under strong alkaline conditions to enhance the catalytic performance [18-20], while most of them are focusing on studying the catalytic performance at low current density and also need high potential to drive the WE. Therefore, it is deserved to develop 3D TMB materials with excellent WE catalytic activity at high current density [21-24].

Mesoporous-based materials are studied for enhancing the WE performance, because it has large specific surface area to expose abundant catalytic activity sites, increase the contact area with electrolyte, and prompt the gas and electrolyte diffusion at high current density [25-29]. Du et al. reported $\mathrm{Co}_{4} \mathrm{~N}-\mathrm{CeO}_{2}$ porous nanosheet self-supported on graphite plate $\left(\mathrm{Co}_{4} \mathrm{~N}-\mathrm{CeO}_{2} / \mathrm{GP}\right)$, which shows low overpotentials for HER $(24 \mathrm{mV})$ and OER $(239 \mathrm{mV})$ at $\pm 10 \mathrm{~mA} \mathrm{~cm}{ }^{-2}$ [30]. It can work at $500 \mathrm{~mA} \mathrm{~cm}^{-2}$ for $50 \mathrm{~h}$ as cathode and anode, exhibiting long-term durability. Ren et al. synthesized ternary 3D $\mathrm{Ni}_{2(1-x)} \mathrm{Mo}_{2 x} \mathrm{P}$ nanowire with mesoporous structure; at -500 and $-1000 \mathrm{~mA} \mathrm{~cm}^{-2}$, it exhibits low overpotentials for HER (240 and $294 \mathrm{mV}$ ) under 1.0 M KOH solution [31]. Although researchers synthesized many mesoporous materials with better electrocatalytic performance, the activity and durability at high current density still cannot meet the demand of industry WE. In addition, most of these catalysts are used only for HER or OER instead of overall water splitting.

In this work, we synthesize a highly efficient $\mathrm{N}$-doped graphene-decorated NiCo alloy coupled with mesoporous $\mathrm{NiCoMoO}$ nano-sheet grown on 3D nickel foam as bifunctional catalyst (NiCo@C-NiCoMoO/NF) for WE. At $\pm 1000 \mathrm{~mA} \mathrm{~cm}^{-2}$, it exhibits excellent catalytic activity with low overpotentials for HER and OER (266 and $390 \mathrm{mV}$ ). More importantly, under 6.0 M KOH solution and $60{ }^{\circ} \mathrm{C}$, it needs ultralow voltage of $1.90 \mathrm{~V}$ to reach $1000 \mathrm{~mA} \mathrm{~cm}^{-2}$ and can maintain for $43 \mathrm{~h}$ as anode and cathode.

\section{Material Synthesis and Characterization}

\subsection{Synthesis of NiCo@C-NiCoMoO/NF Nano-Sheet}

All reagents come from Aladdin Reagent Co., Ltd. without purification. First, nickel foam (NF, $\left.2.0 \times 4.0 \mathrm{~cm}^{2}\right)$ was treated in ethanol, 3.0 M hydrochloric acid, and ultrapure water with ultrasonication, respectively. Second, the cleaned NF was put in an $30 \mathrm{~mL}$ mix solution (ethylene glycol and ultra-pure water) with sodium molybdate dihydrate, urea, and nitrate hexahydrate. Third, the mix solution was put into steel autoclave for $12 \mathrm{~h}$ at $180^{\circ} \mathrm{C}$. After cooling to $25^{\circ} \mathrm{C}$, the NF was washed by ethanol and ultrapure water, and dried overnight under vacuum at $80{ }^{\circ} \mathrm{C}$. Finally, the dried sample was treated at $450{ }^{\circ} \mathrm{C}$ under $5 \%$ $\mathrm{H}_{2}+95 \%$ Ar atmosphere for $2 \mathrm{~h}\left(3{ }^{\circ} \mathrm{C} \mathrm{min}^{-1}\right.$, the obtained sample named as NiCo@C-NiCoMoO/NF), and its mass load on $\mathrm{NF}$ was $\approx 10.5 \mathrm{mg} \mathrm{cm}^{-2}$. Besides, the dried sample was also heated at 350 and $550{ }^{\circ} \mathrm{C}$. NiCo-NiCoMoO/NF nano-sheet was prepared in ultra-pure water with $\mathrm{Ni}, \mathrm{Co}$, and Mo source. 


\subsection{Physical Characterization}

The scanning electron microscopy (SEM, SU8220, HITACHI, Japan) was applied to research the surface morphology of the samples. X-ray diffraction (XRD) was examined on SmartLab, Rigaku Co., D8 Advance (Japan, $\left.\lambda_{(\mathrm{Cu} \mathrm{K \alpha})}=0.15406 \mathrm{~nm}\right)$. Transmission electron microscopy (TEM) and high-resolution TEM (HRTEM) and energydispersive X-ray (EDX) spectroscopy were characterized by a Titan ETEM G2 80-300 (FEI Co., USA). Raman spectroscopy was tested by a Raman spectrometer (Horiba Jobin Yvon Inc., France, $\left.\lambda_{(\mathrm{He} / \mathrm{Ne})}=532 \mathrm{~nm}\right)$. The $\mathrm{N}_{2}$ adsorption/ desorption of the samples at $77 \mathrm{~K}$ was evaluated by the ASAP 2420 instrument (USA, Micrometrics Co.). The elements status of catalyst was tested by X-ray photoelectron spectroscopy (XPS, ESCALab 250Xi, Al X-ray, USA).

\subsection{Electrochemical Tests}

All the electrochemical tests [linear sweep voltammetry (LSV), chronopotentiometry (CP), and electrochemical impedance spectroscopy (EIS)] used the standard threeelectrode system [counter electrode: graphite bar; working electrode: the as-prepared samples (the test area is $0.5 \mathrm{~cm}^{2}$ ); reference electrode: reversible hydrogen electrode] by electrochemical workstation (Germany, ZAHNER) under $1.0 \mathrm{M}$ $\mathrm{KOH}$ solution containing saturated $\mathrm{N}_{2}$. EIS was tested by the three-electrode system from 100,000 to $0.1 \mathrm{~Hz}$; the test potential was -0.2 and $1.5 \mathrm{~V}$ for HER and OER (the amplitude is $5 \mathrm{mV}$ ). The following formula was used for $i R$ correction potential $\left(E_{\text {corr }}\right)$ : (1) $E_{\text {corr }}=E_{\text {mea }}-i R_{\mathrm{s}}$, which was actually measured potential $\left(E_{\text {mea }}\right)$ and the solution resistance $\left(R_{\mathrm{s}}\right)$. Besides, the same condition was used for two-electrode system. The equation [(2) $\eta=b \log |j|+a]$ was used to assess the Tafel plots; the Tafel slope and the current density were $b$ and $j$. The turnover frequency (TOF) and mass activity (MA) of catalyst for HER and OER were calculated based on the reported literatures [32-35].

Besides, $20 \mathrm{wt} \% \mathrm{Pt} / \mathrm{C}$ (anode) and $40 \mathrm{wt} \% \mathrm{IrO}_{2} / \mathrm{C}$ (cathode) were used as noble metal ink (bought from Aladdin with no further treatment). Ethanol $(0.96 \mathrm{~mL})$ and $5.0 \mathrm{wt} \%$ Nafion $(40.0 \mu \mathrm{L})$ mixed solution was applied to disperse this noble metal catalyst; then, it was dropped on $\mathrm{NF}\left(0.5 \mathrm{~cm}^{2}\right)$ and named as $\mathrm{Pt} / \mathrm{C} / \mathrm{NF}$ and $\mathrm{IrO}_{2} / \mathrm{C} / \mathrm{NF}$.

\section{Result and Discussion}

\subsection{Physicochemical Characterization}

$\mathrm{N}$-doped graphene-decorated NiCo alloy coupled with mesoporous NiCoMoO nano-sheet grown on 3D nickel foam was synthesized via the facile two-step methods (Fig. 1).

Figure S1a, b displays the SEM images of NiCo@C$\mathrm{NiCoMoO} / \mathrm{NF}$ (annealed at $450{ }^{\circ} \mathrm{C}$ ), which shows that the nanoparticles are uniformly anchored on the self-supported mesoporous nano-sheet, and it is different from the NiCoMoO nano-sheet precursors with smooth surfaces (Fig. S1c, d). The XRD images in Fig. S2 show that the three diffraction peaks belong to the (111), (200), and (220) planes of $\mathrm{NiCo}$, respectively [36]. Furthermore, the other peaks can be assigned to $\mathrm{Ni}_{2} \mathrm{Mo}_{3} \mathrm{O}_{8}$ (PDF\#37-0855) and $\mathrm{Co}_{2} \mathrm{Mo}_{3} \mathrm{O}_{8}$ (PDF\#34-0511). The XRD results indicate that it composed of NiCo alloy, $\mathrm{Ni}_{2} \mathrm{Mo}_{3} \mathrm{O}_{8}$, and $\mathrm{Co}_{2} \mathrm{Mo}_{3} \mathrm{O}_{8}$.

The TEM, high-angle annular dark field scanning TEM (HAADF-STEM), and high resolution TEM (HRTEM) images (Fig. 2a-g) are used to reveal the lattice fringe,

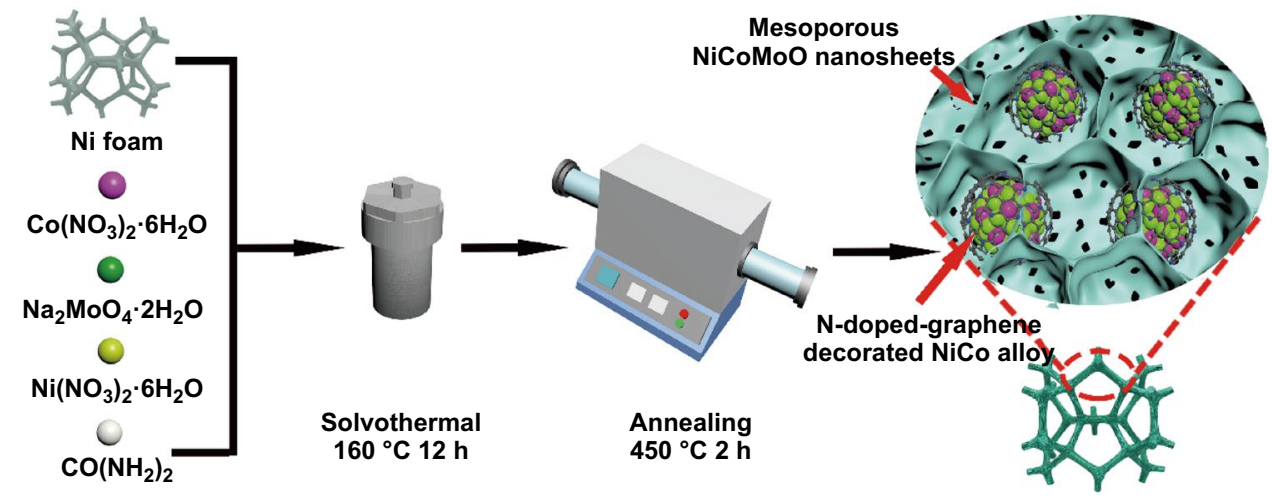

Fig. 1 Schematic for the synthesis of NiCo@C-NiCoMoO/NF 
$\mathrm{N}$-doped graphene, mesoporous nano-sheet, and nanoparticles $(\approx 30-50 \mathrm{~nm})$ structure. The nanoparticles are evenly dispersed on the mesoporous nano-sheet (Fig. 2a). In Fig. 2b-d, the interplanar distances of $0.209 \mathrm{~nm}$ (111), $0.248 \mathrm{~nm}$ (112), and $0.260 \mathrm{~nm}$ (200) are assigned to NiCo, $\mathrm{Ni}_{2} \mathrm{Mo}_{3} \mathrm{O}_{8}$, and $\mathrm{Co}_{2} \mathrm{Mo}_{3} \mathrm{O}_{8}$ (consistent with XRD), respectively [37].

In Fig. 2d, the nanointerface existing between NiCo alloy and $\mathrm{NiCoMoO}$ can facilitate the redistribution of electrons to form the electron-rich and electron-poor species, which can optimize $\mathrm{H}^{*}$ and $\mathrm{H}_{2} \mathrm{O} / \mathrm{OH}^{-}$absorption energy to enhance the performance for HER and OER [22, 38-40]. Furthermore, the NiCo alloy is obviously coated by graphene carbon $(\sim 4$ layers) in Fig. 2e, which can efficiently optimize the distribution of electrons on the catalyst's surface to improve the catalytic activity for WE and prevent the metal dissolution under strong alkaline condition [41].

The graphene carbon of $\mathrm{NiCo} @ \mathrm{C}-\mathrm{NiCoMoO} / \mathrm{NF}$ is also evaluated by Raman in Fig. S3, and the ratio of area D and $\mathrm{G}$ is 1.36 at $450{ }^{\circ} \mathrm{C}$, which is larger than the ones prepared at 350 and $550{ }^{\circ} \mathrm{C}(1.16$ and 1.28), suggesting a larger number of structural defects to enhance the catalytic activity for WE. The EDS elemental mappings (Fig. 2h-m) demonstrate that the $\mathrm{Ni}, \mathrm{Co}, \mathrm{Mo}, \mathrm{O}, \mathrm{C}$, and $\mathrm{N}$ elements are evenly distributed on NiCo@C-NiCoMoO/NF nano-sheet.

Furthermore, it also exhibits mesoporous structure (2-15 nm) in Figs. 2b, c and S4a-c, which can be obtained by HAADF-STEM (Figs. 2f and S4d, e). To further study the mesoporous structure of $\mathrm{NiCo@C-NiCoMoO/NF}$ nano-sheet, the pore volume/size $\left(0.18 \mathrm{~cm}^{3} \mathrm{~g}^{-1} / 6.83 \mathrm{~nm}\right)$ and specific surface area $\left(102.96 \mathrm{~m}^{2} \mathrm{~g}^{-1}\right)$ are characterized by $\mathrm{N}_{2}$ absorption/desorption measurements, and the most parts range of mesoporous peaks is $1.0-14.0 \mathrm{~nm}$ (Fig. S5a, $\mathrm{b}$ and Table S1). This mesoporous nano-sheet possesses a large specific surface area to expose more catalytic active sites and enhance the activity for WE. Additionally, it can increase the contact area with electrolyte to accelerate the release of $\mathrm{H}_{2} / \mathrm{O}_{2}$ bubbles and improve the performance for WE at high current density [26, 28].

Meanwhile, the NiCoMoO nano-sheet precursors also annealed at 350 and $550{ }^{\circ} \mathrm{C}$ to study the effect of post-treatment at different temperatures on the crystal structure, morphology, pore volume/size, and specific surface area (Fig. S5 and Table S1). When the precursors annealed at $350{ }^{\circ} \mathrm{C}$, the XRD peak intensities of $\mathrm{Co}_{2} \mathrm{Mo}_{3} \mathrm{O}_{8}, \mathrm{Ni}_{2} \mathrm{Mo}_{3} \mathrm{O}_{8}$, and $\mathrm{NiCo}$ are too weak (Fig. S2), the nano-sheets are smooth (Fig. S6a, b), and the size of mesoporous is mainly concentrated on $11.1 \mathrm{~nm}$ (Fig. S5c, d). As annealed at $550{ }^{\circ} \mathrm{C}$, the XRD peak intensities of $\mathrm{Co}_{2} \mathrm{Mo}_{3} \mathrm{O}_{8}, \mathrm{Ni}_{2} \mathrm{Mo}_{3} \mathrm{O}_{8}$, and $\mathrm{NiCo}$ are strong, the nano-sheets are broken (Fig. S6c, d), and the material has macroporous structure (Fig. S5e, f). Thus, temperature plays an important effect on the formation of this novel structure.

Subsequently, the electron interaction and elemental status of $\mathrm{Ni}, \mathrm{Co}, \mathrm{Mo}, \mathrm{O}, \mathrm{C}$, and $\mathrm{N}$ elements in NiCo@C$\mathrm{NiCoMoO/NF}$ (Figs. 3 and S7) are proved by XPS. Interestingly, for NiCo@C-NiCoMoO/NF, the Ni $2 p$ peaks of Ni show a $\approx 0.4 \mathrm{eV}$ positive shift compared with that of NiCo$\mathrm{NiCoMoO} / \mathrm{NF}$ (Fig. 3a). For Co $2 p$, the peaks of NiCo@C$\mathrm{NiCoMoO} / \mathrm{NF}$ also show $\mathrm{a} \approx 0.5 \mathrm{eV}$ positive shift as against to that of NiCo-NiCoMoO/NF (Fig. 3b). This is because of different electronegativity between Ni/Co (1.91/1.88), C (2.55), and $\mathrm{N}$ (3.04). So, the $\mathrm{N}$-doped carbon can efficiently optimize the electron structure on the surface of NiCo alloy, which could be beneficial to enhance the performance for WE.

Particularly, the redistribution of electrons could lead to the charge transfer from $\mathrm{NiCo}$ to $\mathrm{N}$-doped carbon, forming electron-rich $\mathrm{N}$-doped graphene and electron-poor NiCo species, which can optimize the absorption energy of $\mathrm{H}^{*}, \mathrm{H}_{2} \mathrm{O}$, and $\mathrm{OH}^{-}$for HER and OER [38, 39]. The high-resolution XPS (HRXPS) spectra of Mo $3 \mathrm{~d}$ are fitted into six mainly peaks at $\mathrm{Mo}^{6+}(235.1 / 232.0), \mathrm{Mo}^{5+}(233.3 / 230.2)$, and $\mathrm{Mo}^{4+}$ (232.5/229.4), respectively (Fig. 3c). Besides, the surface of catalyst is oxidized when exposed to air, resulting in high valence state of $\mathrm{Ni}, \mathrm{Co}$, and $\mathrm{Mo}$ (Fig. 3a-c). As shown in Fig. 3d, the $\mathrm{C} 1 s$ has three peaks at $\mathrm{C}=\mathrm{O}(288.4 \mathrm{eV}), \mathrm{C}-\mathrm{N}$ $(286.2 \mathrm{eV})$, and $\mathrm{C}-\mathrm{C}(284.8 \mathrm{eV})$ that further prove the existence of the $\mathrm{N}$-doped graphene. Moreover, the $\mathrm{N} 1 s$ is located at 401.3 and $398.5 \mathrm{eV}$ in Fig. 3e, assigned to graphitic-N and pyridinic-N, which can result in an important effect on catalytic activity for HER and OER [42]. The peaks of O $1 s$ are absorbed-O (532.4 eV) and NiCoMo-O (530.7 eV) in Fig. 3f, which demonstrate the exist of $\mathrm{Ni}_{2} \mathrm{Mo}_{3} \mathrm{O}_{8}$ and $\mathrm{Co}_{2} \mathrm{Mo}_{3} \mathrm{O}_{8}$.

Therefore, we can draw the concision that the N-doped graphene-decorated NiCo alloy coupled with mesoporous NiCoMoO nano-sheet is successfully prepared. Combining SEM, BET, XRD, and Raman (Figs. S1-S6) results, the following formation mechanism can be proposed: different adsorption enthalpies of $\mathrm{Ni}, \mathrm{Co}$, and Mo can lead to the part of $\mathrm{Ni}$ and $\mathrm{Co}$ atoms segregated from precursors to form NiCo alloy [43, 44]. When the precursors annealed at 

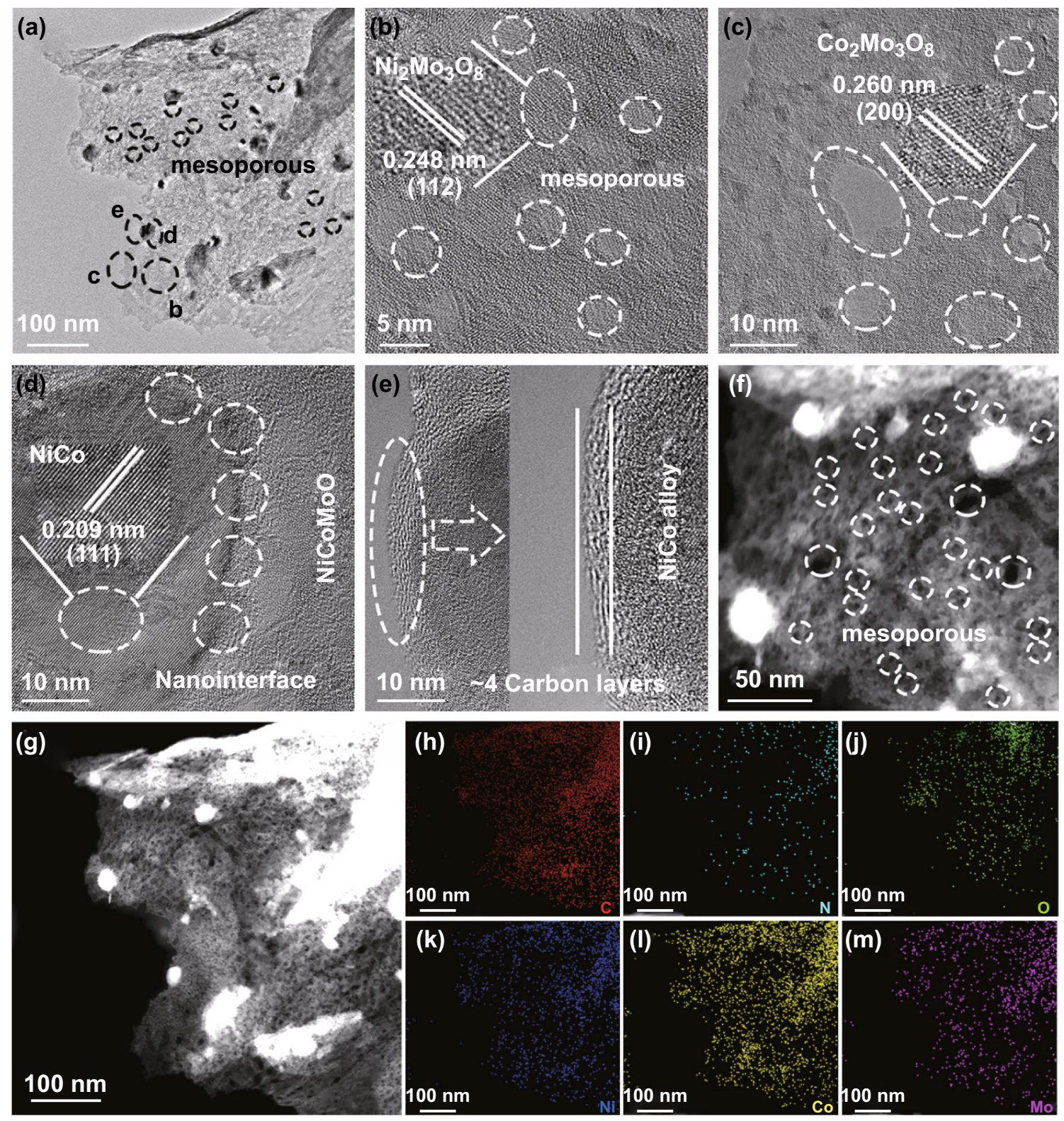

Fig. 2 a-e TEM and HRTEM, f-m HAADF-STEM images and EDS elemental mappings of C, N, O, Ni, Co, and Mo of NiCo@C-NiCoMoO/ $\mathrm{NF}$

$450{ }^{\circ} \mathrm{C}$, parts of $\mathrm{Ni}$ and $\mathrm{Co}$ combine with $\mathrm{O}$ and $\mathrm{Mo}$ to form $\mathrm{Ni}_{2} \mathrm{Mo}_{3} \mathrm{O}_{8}$ and $\mathrm{Co}_{2} \mathrm{Mo}_{3} \mathrm{O}_{8}$, which can form the heterojunction between $\mathrm{NiCo}$ and $\mathrm{NiCoMoO}$ [37]. Besides, NiCo alloy can catalyze the organic carbon to form $\mathrm{N}$-doped graphene [45]. The formation of mesoporous is caused by the dehydration from the precursors during the high-temperature calcination process, and the pore size is related to the temperature. However, when the precursors are annealed at $350{ }^{\circ} \mathrm{C}$, the NiCo alloy cannot be reduced from the precursors, and the surface organic carbon cannot form more N-doped graphene. This will decrease the intrinsic activity for WE. It can also be seen from SEM and BET pictures (Figs. S6a, b and S5c, d), the material cannot be dehydrated to form a mesoporous nano-sheet structure due to the low temperature that cannot provide enough specific surface area for exposing more active sites. When the precursors annealing at $550{ }^{\circ} \mathrm{C}$, the $\mathrm{Ni}$ and $\mathrm{Co}$ atoms will be quickly reduced to form strong NiCo alloy and $\mathrm{NiCoMoO}$ (Fig. S2), and the 

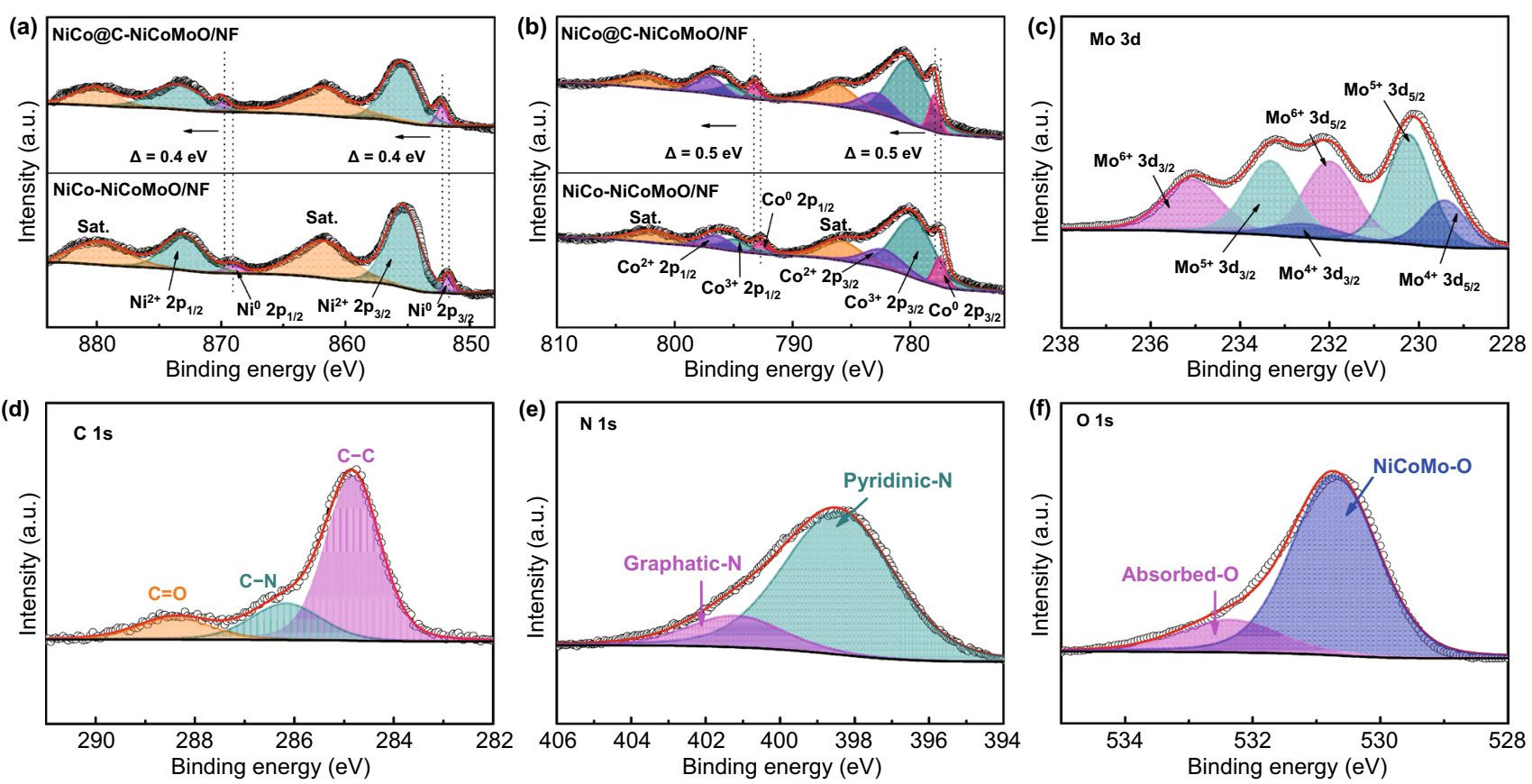

Fig. 3 a, b High-resolution XPS spectra of Ni $2 p$ and Co $2 p$ for NiCo@C-NiCoMoO/NF and NiCo-NiCoMoO/NF. c Mo $3 d$, d C $1 s$, e N $1 s$, and f O $1 s$ of NiCo@C-NiCoMoO/NF

nano-sheet is quickly dehydrated to form the macroporous structure and almost broken as nanoparticles (Fig. S6c, d). It will lower the intrinsic activity for WE and not provide large specific surface area for exposing more active sites. In summary, the $\mathrm{N}$-doped graphene-decorated $\mathrm{NiCo}$ alloy, mesoporous $\mathrm{NiCoMoO}$ nano-sheet, and heterostructures are formed at $450{ }^{\circ} \mathrm{C}$, which have the highest intrinsic activity and specific surface area. The heterostructures owe good electrochemical activity for HER and OER that is confirmed by LSV, Tafel, and EIS characterization (Figs. S11 and S22).

\subsection{HER Catalytic Performance of NiCo@C-NiCoMoO/NF}

The HER electrocatalytic activity of the samples is evaluated by a three-electrode system under $1.0 \mathrm{M} \mathrm{KOH}$ solution containing saturated $\mathrm{N}_{2}$. Obviously, the $\mathrm{NiCo@C-NiCoMoO/}$ $\mathrm{NF}$ only acquires low overpotentials of 39 and $266 \mathrm{mV}$ at -10 and $-1000 \mathrm{~mA} \mathrm{~cm}{ }^{-2}$ (Figs. $4 \mathrm{a}$ and $\mathrm{S} 8$ ), which is lower than that of NiCo-NiCoMoO/NF $\left(\eta_{-10}=75 \mathrm{mV}\right.$; $\left.\eta_{-1000}=303 \mathrm{mV}\right)$. Thus, the HER activity of NiCo@C$\mathrm{NiCoMoO} / \mathrm{NF}$ is significantly improved after the NiCo alloy coated by $\mathrm{N}$-doped graphene, especially at high current density, which could be attributed to the $\mathrm{N}$-doped graphene structure, optimized the surface electronic distribution, and improved the activity and conductivity of catalysts. Furthermore, the overpotentials of NiCo@C-NiCo$\mathrm{MoO} / \mathrm{NF}$ are lower than that of precursors $\left(\eta_{-10}=141 \mathrm{mV}\right.$; $\left.\eta_{-1000}=443 \mathrm{mV}\right), \mathrm{NF}\left(\eta_{-10}=223 \mathrm{mV} ; \eta_{-1000}=561 \mathrm{mV}\right)$ and closed to Pt/C/NF $\left(\eta_{-10}=30 \mathrm{mV} ; \eta_{-1000}=231 \mathrm{mV}\right)$. The overpotential at $-1000 \mathrm{~mA} \mathrm{~cm}^{-2}$ is better than most of the reported literatures (Fig. 4b), which indicates that it could meet the demand of catalytic activity at high current density for industrial-scale.

Figure S9 displays the LSV curves of NiCo@C-NiCo$\mathrm{MoO} / \mathrm{NF}$ with/without $i R$ correction for HER. Tafel slope obtained from LSV curve is carried out to further research the kinetic of HER (Fig. 4c). It shows that Tafel slope of $\mathrm{NiCo} @ \mathrm{C}-\mathrm{NiCoMoO} / \mathrm{NF}$ is only $63.50 \mathrm{mV} \mathrm{dec}{ }^{-1}$, outperforming NiCo-NiCoMoO/NF $\left(98.62 \mathrm{mV} \mathrm{dec}^{-1}\right)$, precursors $\left(117.19 \mathrm{mV} \mathrm{dec}^{-1}\right), \mathrm{NF}\left(159.99 \mathrm{mV} \mathrm{dec}^{-1}\right)$, and similar with $\mathrm{Pt} / \mathrm{C} / \mathrm{NF}\left(42.24 \mathrm{mV} \mathrm{dec}^{-1}\right)$. The smaller value of Tafel suggests that NiCo@C-NiCoMoO/NF can readily overcome the kinetics process of HER. As shown in Table S3, TOF and MA values of NiCo@C-NiCoMoO/NF at the overpotentials of $50,100,150$, and $200 \mathrm{mV}$ also indicate its high catalytic activity for HER and better than most reported results in the literatures (Table S4). 
The EIS is used to estimate the kinetics of HER. In Fig. $\mathrm{S} 10$, it displays that $\mathrm{NiCo} @ \mathrm{C}-\mathrm{NiCoMoO} / \mathrm{NF}$ has the smallest charge transfer resistance $\left(R_{\mathrm{ct}}\right)$ compared with another samples, revealing its best electron transfer rate. For precursors annealed at different temperatures, the LSV curves, Tafel slope, and EIS of HER are displayed in Fig. S11, which demonstrate that the precursors annealing at $450{ }^{\circ} \mathrm{C}$ exhibits the best activity.

The electrochemical active surface area (EASA) is evaluated by the double-layer capacitance $\left(C_{\mathrm{dl}}\right)$ that is obtained by cyclic voltammetry $(\mathrm{CV})$ methods under no Faradic regions (Fig. S12). NiCo@C-NiCoMoO/NF has the largest $C_{\mathrm{dl}}$ value $\left(28.81 \mathrm{mF} \mathrm{cm}^{-2}\right)$; it is better than $\mathrm{NiCo}-\mathrm{NiCoMoO} / \mathrm{NF}$ $\left(17.60 \mathrm{mF} \mathrm{cm}^{-2}\right)$, indicating that $\mathrm{N}$-doped carbon-decorated NiCo alloy can effectively boost the intrinsic activity and speed up the HER process. The LSV curves are normalized by EASA in Fig. S13; apparently, the intrinsic catalytic activity of $\mathrm{NiCo@C-NiCoMoO/NF}$ is better than NiCo-NiCoMoO/NF.

In Fig. 4d, we research the HER durability of NiCo@CNiCoMoO/NF under 1.0 M KOH solution by $\mathrm{CP}$ measurement at $-1000 \mathrm{~mA} \mathrm{~cm}{ }^{-2}$, which displays excellent stability after continuous work $340 \mathrm{~h}$, and the potential has only changed $18 \mathrm{mV}$. Furthermore, the value of overpotential at $-1000 \mathrm{~mA} \mathrm{~cm}^{-2}$ and $R_{\mathrm{ct}}$ at $-0.2 \mathrm{~V}$ ( $v s$ RHE) after stability test is negligibly changed, indicating its good stability. The SEM (Fig. S15) and HRTEM (Fig. S16) images of NiCo@C$\mathrm{NiCoMoO/NF}$ after HER stability test showed that it maintains the pristine morphology. In addition, the HRXPS (Fig. S17) spectra of Mo, Ni, and Co for NiCo@C-NiCoMoO/ NF show no obvious change. These results confirm that the NiCo@C-NiCoMoO/NF exhibits an outstanding HER durability in $1.0 \mathrm{M} \mathrm{KOH}$ solution. The reason could be due to that the $\mathrm{N}$-doped graphene-decorated NiCo alloy framework can prevent metal from dissolving in the strong alkaline solution, thus improving the chemical stability. Furthermore, the selfsupporting mesoporous nano-sheet has large specific surface area to increase wettability of the catalyst, facilitate the release of $\mathrm{H}_{2}$ bubbles, avoid the use of binder to improve the electron transfer efficiency, and prevent the active substance from spalling to enhance the mechanical stability.
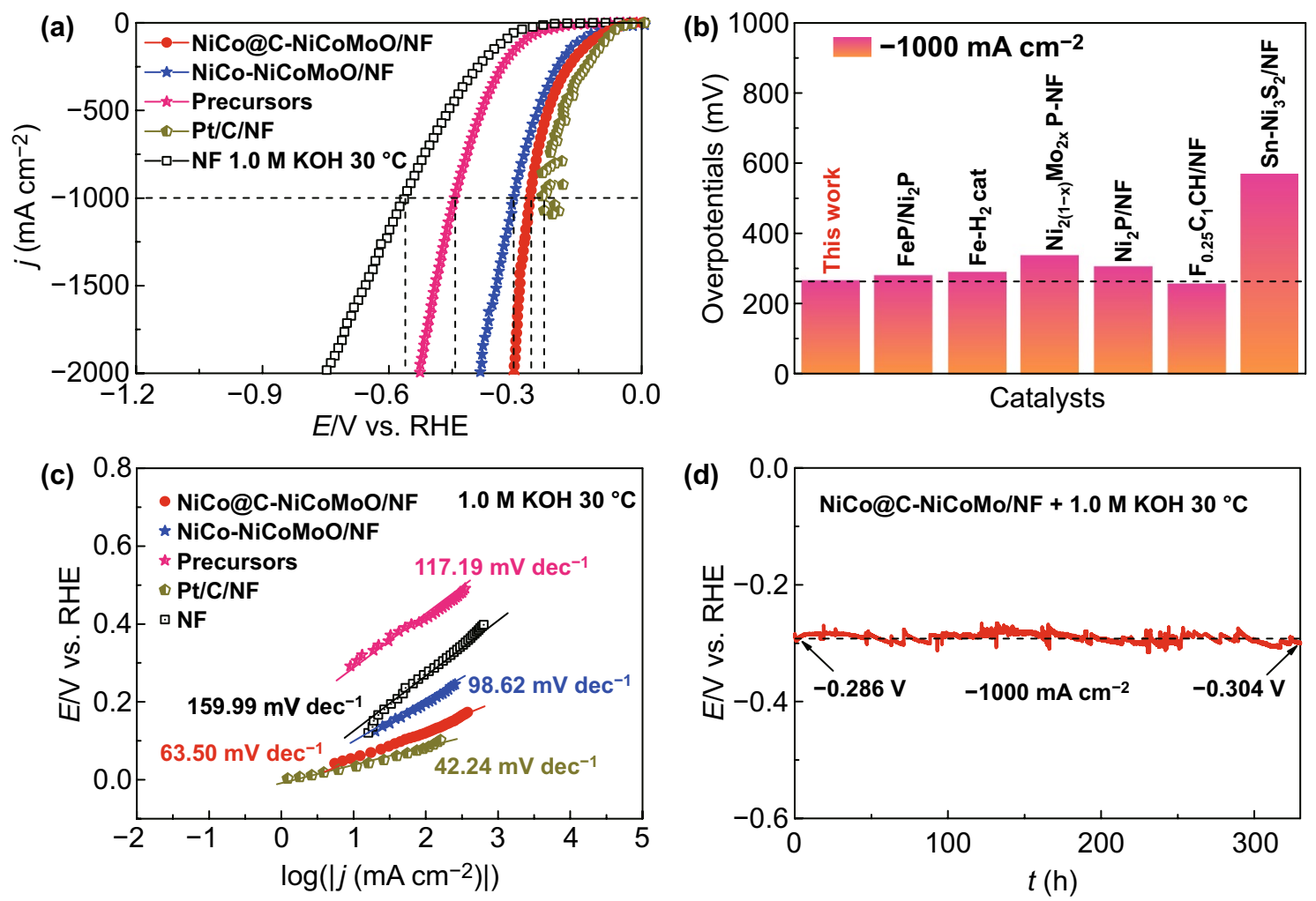

Fig. 4 a LSV curves of HER for investigated samples. b Comparisons of HER overpotentials in this work and reported catalysts [31, 47-52]. c Corresponding Tafel curves. d CP curve 


\subsection{OER Catalytic Performance of NiCo@C-NiCoMoO/NF}

We evaluate OER catalytic performance of NiCo@C-NiCo$\mathrm{MoO} / \mathrm{NF}$ under the same solution. Figures $5 \mathrm{a}$ and $\mathrm{S} 18$ show their $i R$ corrected LSV curves. Similar to HER performance, NiCo@C-NiCoMoO/NF has the low overpotentials (260 and $390 \mathrm{mV}$ ) at 10 and $1000 \mathrm{~mA} \mathrm{~cm}{ }^{-2}$, which is smaller than that of NiCo-NiCoMoO/NF (280 and $459 \mathrm{mV}$ ), indicating that the $\mathrm{N}$-doped graphene can efficiently adjust the surface electronic of the catalyst to enhance the intrinsic activity of catalyst. In addition, it outperforms precursors $\left(\eta_{10}=320 \mathrm{mV} ; \eta_{1000}=554 \mathrm{mV}\right), \mathrm{IrO}_{2} / \mathrm{C} / \mathrm{NF}\left(\eta_{10}=290 \mathrm{mV}\right.$; $\left.\eta_{1000}=476 \mathrm{mV}\right)$, and NF $\left(\eta_{10}=340 \mathrm{mV} ; \eta_{1000}=624 \mathrm{mV}\right)$. Importantly, the overpotential at $1000 \mathrm{~mA} \mathrm{~cm}^{-2}$ is better than most of the reported literatures as shown in Fig. 5b. Furthermore, the LSV curves of OER for NiCo@C-NiCo$\mathrm{MoO} / \mathrm{NF}$ with/without $i R$ correction are shown in Fig. S19, and the LSV curves of OER for NiCo@C-NiCoMoO/NF and
NiCo-NiCoMoO/NF are normalized by EASAs in Fig. S20 similar to HER performance.

We obtain the Tafel slopes from the LSV curves to evaluate the kinetics of OER (Fig. 5c). Obviously, the Tafel slopes are decreased in following order: NF $\left(170.19 \mathrm{mV} \mathrm{dec}^{-1}\right)>$ precursors $\left(155.56 \mathrm{mV} \mathrm{dec}^{-1}\right)>\mathrm{NiCo}-$ $\mathrm{NiCoMoO} / \mathrm{NF} \quad\left(113.13 \mathrm{mV} \quad \mathrm{dec}^{-1}\right)>\mathrm{IrO}_{2} / \mathrm{C} /$ $\mathrm{NF}\left(94.65 \mathrm{mV} \mathrm{dec}^{-1}\right)>\mathrm{NiCo} @ \mathrm{C}-\mathrm{NiCoMoO} / \mathrm{NF}$ $\left(75.15 \mathrm{mV} \mathrm{dec}^{-1}\right)$. The EIS is used to estimate the electrode kinetics of OER. Figure S21 displays that NiCo@C-NiCo$\mathrm{MoO} / \mathrm{NF}$ has the smallest $R_{\mathrm{ct}}$ compared with another samples, revealing it possesses the best electron transfer rate. The above results also suggest that $\mathrm{N}$-doped graphene-decorated $\mathrm{NiCo}$ alloy coupled with mesoporous $\mathrm{NiCoMoO}$ nano-sheet can effectively speed up the OER process. Additionally, in Fig. S22, the catalytic activity is different when precursors annealed at 350,450 , and $550{ }^{\circ} \mathrm{C}$, and the precursors reducing at $450{ }^{\circ} \mathrm{C}$ exhibit the best activity. Subsequently, the calculated TOF and MA values of $\mathrm{NiCo} @ \mathrm{C}-\mathrm{NiCoMoO} / \mathrm{NF}$
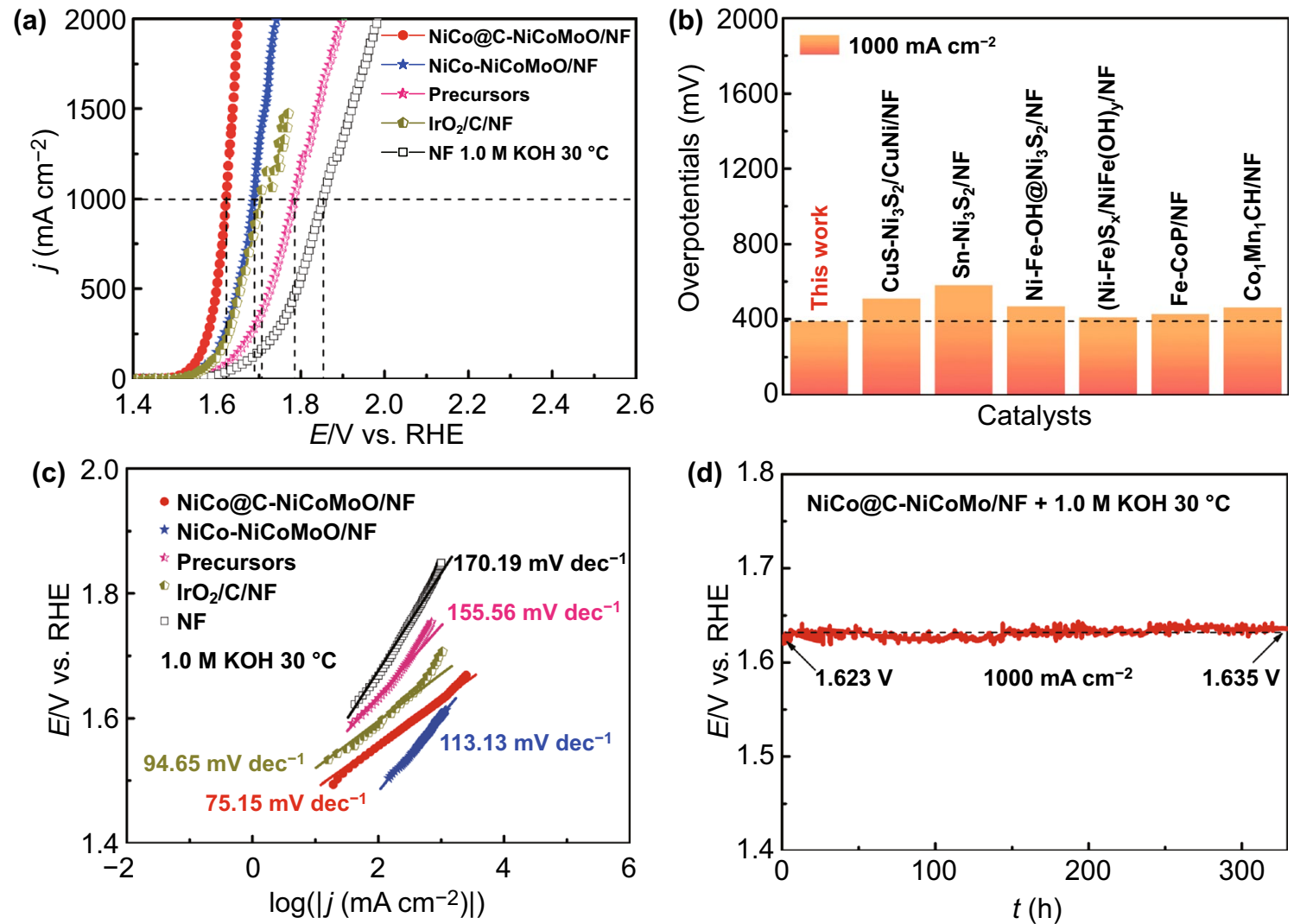

Fig. 5 a LSV curves of OER for investigated samples. b Comparisons of OER overpotentials in this work and reported catalysts [8, 47, 50, 53-56]. c Corresponding Tafel curves. d CP curve 
for OER (Table S5) display its fast OER kinetics, which is higher than most reported literatures (Table S6).

The stability is also essential to evaluate the performance of catalyst, especially at high current density. As shown in Fig. 5d, NiCo@C-NiCoMoO/NF can keep for $340 \mathrm{~h}$ at $1000 \mathrm{~mA} \mathrm{~cm}^{-2}$, and the change of potential is $12 \mathrm{mV}$, displaying an outstanding stability. Furthermore, we also study the catalytic activity after stability test by LSV and EIS curves (Fig. S23), and it shows ignorable change. The outstanding durability of NiCo@C-NiCo$\mathrm{MoO} / \mathrm{NF}$ could be assigned to the $\mathrm{N}$-doped graphenedecorated NiCo alloy, which can avoid corrosion in the harsh alkaline environment, thus improving the chemical stability. Besides, the self-supporting mesoporous nanosheet can enhance the mechanical stability, since it has large specific surface area to increase the contact area with electrolyte and prompt the release of $\mathrm{O}_{2}$ bubbles.

After the durability tests, SEM images of NiCo@C$\mathrm{NiCoMoO/NF}$ maintain the pristine morphology (Fig. S24), and HRTEM images show that the mesoporous nano-sheet structure keeps well (Fig. S25), suggesting its excellent stability. In addition, the peak of the $\mathrm{Ni}^{0}$ and $\mathrm{Co}^{0}$ disappeared (Fig. S26a, b), which indicates that the surface of catalyst is oxidized during the OER process, (a)

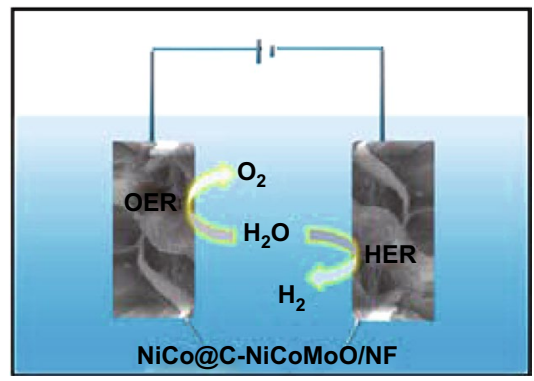

(c)

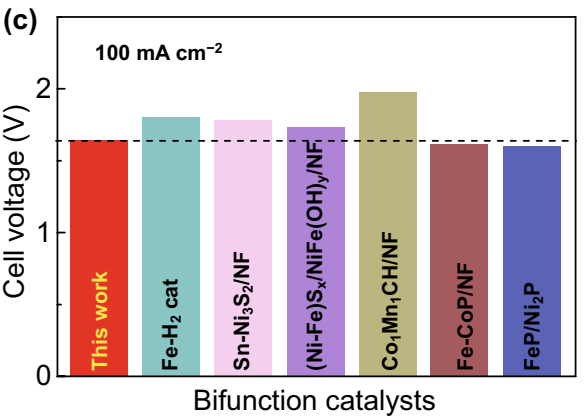

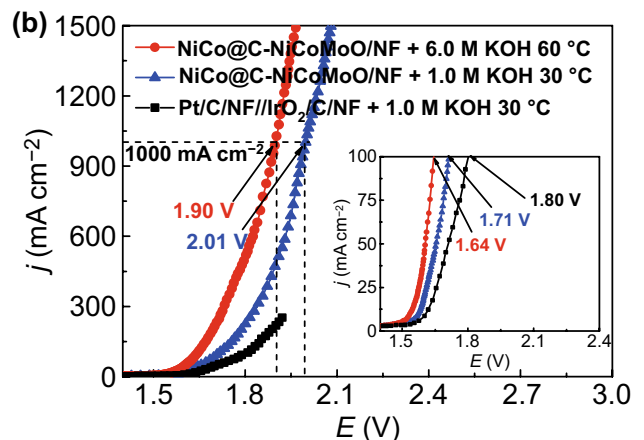

(d) 3.0

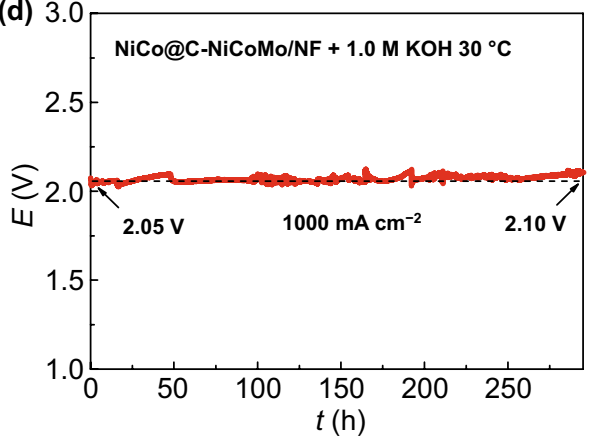

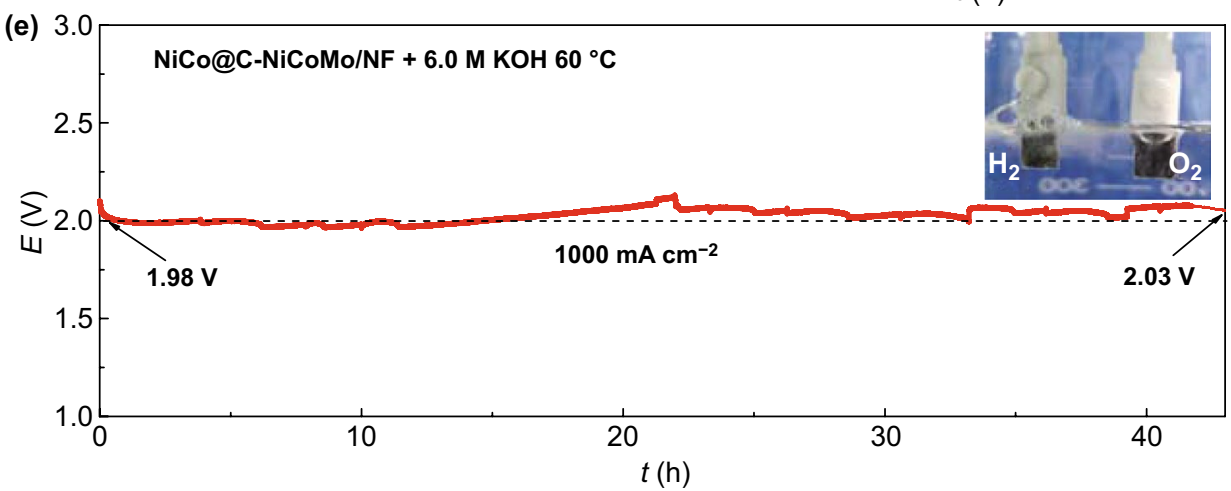

Fig. 6 a Using NiCo@ C-NiCoMoO/NF as the cathode and anode for water electrolysis. b LSV curves of OER for the investigated samples. c Comparison WE potentials for this work and reported catalysts $[8,47,51,53,55,57]$. d, e CP curves of NiCo@ C-NiCoMoO/NF under 1.0 M $\mathrm{KOH}+30{ }^{\circ} \mathrm{C}$ and $6.0 \mathrm{M} \mathrm{KOH}+60{ }^{\circ} \mathrm{C}$ 
and it could form the $\mathrm{Ni} / \mathrm{CoOOH}$ [46]. As displayed in Fig. S26c, the XPS spectra of $\mathrm{Mo}^{4+}$ are also oxidized to $\mathrm{Mo}^{6+}$ and $\mathrm{Mo}^{5+}$, further suggesting the surface oxidation.

\subsection{WE Catalytic Performance of NiCo@C-NiCoMoO/ NF}

Based on the excellent performance of $\mathrm{NiCo} @ \mathrm{C}-\mathrm{NiCoMoO} /$ NF toward HER and OER in alkaline solution, the two-electrode system is used to evaluate the WE performance by using it as bifunctional catalyst (Fig. 6a).

In Fig. 6b, at $100 \mathrm{~mA} \mathrm{~cm}^{-2}$, under $1.0 \mathrm{M} \mathrm{KOH}$ solution at $30{ }^{\circ} \mathrm{C}$, WE performance of NiCo@C-NiCoMoO/ $\mathrm{NF}(1.71 \mathrm{~V})$ is better than that of the $\mathrm{Pt} / \mathrm{C} / \mathrm{NF} \| \mathrm{IrO}_{2} / \mathrm{C} / \mathrm{NF}$ $(1.80 \mathrm{~V})$ couple; it is smaller than most of the reported datum as shown in Fig. 6c. Interestingly, the NiCo@C$\mathrm{NiCoMoO} / \mathrm{NF}$ only requires a low potential of $2.01 \mathrm{~V}$ to deliver $1000 \mathrm{~mA} \mathrm{~cm}^{-2}$ and perform for $295 \mathrm{~h}$ with negligible change $(50 \mathrm{mV}$, Fig. $6 \mathrm{~d})$, indicating that it is promising for industrial hydrogen production.

Additionally, as exhibited in Fig. S27, the amount of $\mathrm{H}_{2}$ and $\mathrm{O}_{2}$ is acquired by water drainage method at $0,25,50,75,100$, and $125 \mathrm{~min}$ operating at $\pm 10.0 \mathrm{~mA}$. Figure S27a, b shows the volume ratio of $\mathrm{H}_{2}$ and $\mathrm{O}_{2}$ is about $2: 1$, which is consistent with theoretical values, suggesting the closely $100 \%$ Faradic efficiency for WE.

Subsequently, NiCo@C-NiCoMoO/NF is tested in $6.0 \mathrm{M} \mathrm{KOH}+60{ }^{\circ} \mathrm{C}$ (Fig. 6b, e); it only needs $1.90 \mathrm{~V}$ at $1000 \mathrm{~mA} \mathrm{~cm}^{-2}$ and can keep for $43 \mathrm{~h}$ without obvious attenuation. Therefore, NiCo@C-NiCoMoO/NF with excellent performance provides a promising material for WE to hydrogen production.

\section{Conclusions}

In summary, NiCo@ $\mathrm{C}-\mathrm{NiCoMoO} / \mathrm{NF}$, a unique $\mathrm{N}$-doped graphene-encapsulated structure and self-supported mesoporous nano-sheet, is prepared by solvothermal method and annealing treatment. As bifunctional catalyst, it displays outstanding HER and OER performance in $1.0 \mathrm{M} \mathrm{KOH}$ solution, which only needs overpotentials of 266 and $390 \mathrm{mV}$ at $\pm 1000 \mathrm{~mA} \mathrm{~cm}^{-2}$, and shows superior stability for $340 \mathrm{~h}$ with no evident activity decrease. More importantly, when applied as anode and cathode in $6.0 \mathrm{M} \mathrm{KOH}+60{ }^{\circ} \mathrm{C}$, it exhibits a low potential of $1.90 \mathrm{~V}$ at $1000 \mathrm{~mA} \mathrm{~cm}^{-2}$ and can work for $43 \mathrm{~h}$ without obvious attenuation, exhibiting performance close to actual application. Therefore, this work may provide a promising catalyst with high catalytic activity and stability for industrial water electrolysis.

Acknowledgements This work is supported by the National Natural Science Foundation of China (21872040), the Hundred Talents Program of Guangxi Universities, and the Excellent Scholars and Innovation Team of Guangxi Universities.

Open Access This article is licensed under a Creative Commons Attribution 4.0 International License, which permits use, sharing, adaptation, distribution and reproduction in any medium or format, as long as you give appropriate credit to the original author(s) and the source, provide a link to the Creative Commons licence, and indicate if changes were made. The images or other third party material in this article are included in the article's Creative Commons licence, unless indicated otherwise in a credit line to the material. If material is not included in the article's Creative Commons licence and your intended use is not permitted by statutory regulation or exceeds the permitted use, you will need to obtain permission directly from the copyright holder. To view a copy of this licence, visit http://creativecommons.org/licenses/by/4.0/.

Supplementary Information The online version contains supplementary material available at https://doi.org/10.1007/s4082 0-021-00607-5.

\section{References}

1. A. Hossain, K. Sakthipandi, A.K.M.A. Ullah, S. Roy, Recent progress and approaches on carbon-free energy from water splitting. Nano-Micro Lett. 11, 103 (2019). https://doi. org/10.1007/s40820-019-0335-4

2. S.S. Chen, T. Hisatomi, G.J. Ma, Z. Wang, Z.H. Pan et al., Metal selenides for photocatalytic Z-scheme pure water splitting mediated by reduced graphene oxide. Chin. J. Catal. 40, 1668-1672 (2019). https://doi.org/10.1016/s1872 -2067(19)63326-7

3. P.W. Menezes, A. Indra, I. Zaharieva, C. Walter, S. Loos et al., Helical cobalt borophosphates to master durable overall watersplitting. Energy Environ. Sci. 12, 988-999 (2019). https://doi. org/10.1039/c8ee01669k

4. T.Q. Yu, Q.L. Xu, G.F. Qian, J.L. Chen, H. Zhang et al., Amorphous $\mathrm{CoO}_{\mathrm{x}}$-decorated crystalline $\mathrm{RuO}_{2}$ nanosheets as bifunctional catalysts for boosting overall water splitting at large current density. ACS Sustain. Chem. Eng. 8, 1752017526 (2020). https://doi.org/10.1021/acssuschemeng.0c067 82

5. X. Luo, P.X. Ji, P.Y. Wang, R.L. Cheng, D. Chen et al., Interface engineering of hierarchical branched Mo-doped $\mathrm{Ni}_{3} \mathrm{~S}_{2} /$ $\mathrm{Ni}_{\mathrm{x}} \mathrm{P}_{\mathrm{y}}$ hollow heterostructure nanorods for efficient overall 
water splitting. Adv. Energy Mater. 10, 1903891 (2020). https ://doi.org/10.1002/aenm.201903891

6. S.J. Deng, K.L. Zhang, D. Xie, Y. Zhang, Y.Q. Zhang et al., Hig-index-faceted $\mathrm{Ni}_{3} \mathrm{~S}_{2}$ branch arrays as bifunctional electrocatalysts for efficient water splitting. Nano-Micro Lett. 11, 12 (2019). https://doi.org/10.1007/s40820-019-0242-8

7. Z. Qiu, C.W. Tai, G.A. Niklasson, T. Edvinsson, Direct observation of active catalyst surface phases and the effect of dynamic self-optimization in NiFe-layered double hydroxides for alkaline water splitting. Energy Environ. Sci. 12, 572-581 (2019). https://doi.org/10.1039/c8ee03282c

8. Q.J. Che, Q. Li, Y. Tan, X.H. Chen, X. Xu et al., One-step controllable synthesis of amorphous $(\mathrm{Ni}-\mathrm{Fe}) \mathrm{S} / \mathrm{NiFe}(\mathrm{OH})$ hollow microtube/sphere films as superior bifunctional electrocatalysts for quasi-industrial water splitting at large-current-density. Appl. Catal. B 246, 337-348 (2019). https://doi. org/10.1016/j.apcatb.2019.01.082

9. P. Babar, A. Lokhande, H.H. Shin, B. Pawar, M.G. Gang et al., Cobalt iron hydroxide as a precious metal-free bifunctional electrocatalyst for efficient overall water splitting. Small 14, 1702568 (2018). https://doi.org/10.1002/smll.201702568

10. T. Ouyang, X.T. Wang, X.Q. Mai, A.N. Chen, Z.Y. Tang et al., Coupling magnetic single-crystal $\mathrm{Co}_{2} \mathrm{Mo}_{3} \mathrm{O}_{8}$ with ultrathin nitrogen-rich carbon layer for oxygen evolution reaction. Angew. Chem. Int. Ed. 59, 11948-11957 (2020). https://doi. org/10.1002/ange.202004533

11. H.T. Liu, J.Y. Guan, S.X. Yang, Y.H. Yu, R. Shao et al., Metalorganic framework-derived $\mathrm{Co}_{2} \mathrm{P}$ nanoparticle/multi-doped porous carbon as a trifunctional electrocatalyst. Adv. Mater. 32, 2003649 (2020). https://doi.org/10.1002/adma.202003649

12. J.W. Li, W.M. Xu, J.X. Luo, D. Zhou, D.W. Zhang et al., Synthesis of 3D hexagram-like cobalt-manganese sulfides nanosheets grown onnickel foam: a bifunctional electrocatalyst for overall water splitting. Nano-micro Lett. 10, 6 (2018). https://doi.org/10.1007/s40820-017-0160-6

13. G.B. Darband, M. Aliofkhazraei, S. Hyun, A.S. Rouhaghdam, S. Shanmugam, Electrodeposited Ni-Co-P hierarchical nanostructure as a cost-effective and durable electrocatalyst with superior activity for bifunctional water splitting. J. Power Sources 429, 156-167 (2019). https://doi.org/10.1016/j.jpows our.2019.04.050

14. S. Anantharaj, S. Chatterjee, K.C. Swaathini, T.S. Amarnath, E. Subhashini et al., Stainless steel scrubber: a cost efficient catalytic electrode for full water splitting in alkaline medium. ACS Sustain. Chem. Eng. 6, 2498-2509 (2018). https://doi. org/10.1021/acssuschemeng.7b03964

15. J.J. Lu, S.B. Yin, P.K. Shen, Carbon-encapsulated electrocatalysts for the hydrogen evolution reaction. Electrochem. Energy Rev. 2, 105-127 (2018). https://doi.org/10.1007/s4191 8-018-0025-9

16. Y.C. Tu, P.J. Ren, D.H. Deng, X.H. Bao, Structural and electronic optimization of graphene encapsulating binary metal for highly efficient water oxidation. Nano Energy 52, 494-500 (2018). https://doi.org/10.1016/j.nanoen.2018.07.062

17. C.Y. Chen, S.L. Liu, X.Q. Mu, R.L. Cheng, S.Y. Lin et al., In situ engineering of hollow porous $\mathrm{Mo}_{2} \mathrm{C} @ \mathrm{C}$ nanoballs derived from giant Mo-polydopamine clusters as highly efficient electrocatalysts for hydrogen evolution. Front. Chem. 8, 170 (2020). https://doi.org/10.3389/fchem.2020.00170

18. J.H. Cao, K.X. Wang, J.Y. Chen, C.J. Lei, B. Yang et al., Nitrogen-doped carbon-encased bimetallic selenide for high-performance water electrolysis. Nano-Micro Lett. 11, 67 (2019). https://doi.org/10.1007/s40820-019-0299-4

19. G.F. Qian, J.L. Chen, L. Luo, T.Q. Yu, Y.M. Wang et al., Industrially promising nanowire heterostructure catalyst for enhancing overall water splitting at large current density. ACS Sustain. Chem. Eng. 8, 12063-12071 (2020). https:// doi.org/10.1021/acssuschemeng.0c03263

20. J. Balamurugan, T.T. Nguyen, V. Aravindan, N.H. Kim, J.H. Lee, Highly reversible water splitting cell building from hierarchical 3D nickel manganese oxyphosphide nanosheets. Nano Energy 69, 104432 (2020). https://doi.org/10.1016/j.nanoe n.2019.104432

21. S. Niu, W.J. Jiang, T. Tang, L.P. Yuan, H. Luo et al., Autogenous growth of hierarchical $\mathrm{NiFe}(\mathrm{OH})_{\mathrm{x}} / \mathrm{FeS}$ nanosheet-onmicrosheet arrays for synergistically enhanced high-output water oxidation. Adv. Funct. Mater. 29, 1902180 (2019). https ://doi.org/10.1002/adfm.201902180

22. J.X. Yuan, X.D. Cheng, H.Q. Wang, C.J. Lei, S. Pardiwala et al., A superaerophobic bimetallic selenides heterostructure for efficient industrial-level oxygen evolution at ultra-high current densities. Nano-Micro Lett. 12, 104 (2020). https://doi. org/10.1007/s40820-020-00442-0

23. S. Xue, Z.B. Liu, C.Q. Ma, H.M. Cheng, W.C. Ren, A highly active and durable electrocatalyst for large current density hydrogen evolution reaction. Sci. Bull. 65, 123-130 (2020). https://doi.org/10.1016/j.scib.2019.10.024

24. J.U. Yang, A.R. Mohmad, Y. Wang, R. Fullon, X.J. Song et al., Ultrahigh-current-density niobium disulfide catalysts for hydrogen evolution. Nat. Mater. 18, 1309-1314 (2019). https://doi.org/10.1038/s41563-019-0463-8

25. A. Saad, H.J. Shen, Z.X. Cheng, R. Arbi, B.B. Guo et al., Mesoporous ternary nitrides of earth-abundant metals as oxygen evolution electrocatalyst. Nano-Micro Lett. 12, 79 (2020). https://doi.org/10.1007/s40820-020-0412-8

26. Y. Li, B. Wei, Z.P. Yu, O. Bondarchuk, A. Araujo et al., Bifunctional porous cobalt phosphide foam for high-currentdensity alkaline water electrolysis with 4000-h long stability. ACS Sustain. Chem. Eng. 8, 10193-10200 (2020). https://doi. org/10.1021/acssuschemeng.0c02671

27. S.C. Zhang, W.B. Wang, F.L. Hu, Y. Mi, S.Z. Wang et al., 2D $\mathrm{CoOOH}$ sheet-encapsulated $\mathrm{Ni}_{2} \mathrm{P}$ into tubular arrays realizing $1000 \mathrm{~mA} \mathrm{~cm}^{-2}$-level-current-density hydrogen evolution over $100 \mathrm{~h}$ in neutral water. Nano-Micro Lett. 12, 140 (2020). https ://doi.org/10.1007/s40820-020-00476-4

28. S.S. Wang, Y.C. Xu, R.R. Fu, H.H. Zhu, Q.Z. Jiao et al., Rational construction of hierarchically porous $\mathrm{Fe}-\mathrm{Co} / \mathrm{N}$-doped carbon/rGO composites for broadband microwave absorption. Nano-Micro Lett. 11, 76 (2019). https://doi.org/10.1007/s4082 0-019-0307-8

29. A. Kumar, S. Bhattacharyya, Porous NiFe-oxide nanocubes as bifunctional electrocatalysts for efficient water-splitting. ACS 
Appl. Mater. Interfaces 9, 41906-41915 (2017). https://doi. org/10.1021/acsami.7b14096

30. H.M. Sun, C.Y. Tian, G.L. Fan, J.N. Qi, Z.T. Liu et al., Boosting activity on $\mathrm{Co}_{4} \mathrm{~N}$ porous nanosheet by coupling $\mathrm{CeO}_{2}$ for efficient electrochemical overall water splitting at high current densities. Adv. Funct. Mater. 30, 1910596 (2020). https://doi. org/10.1002/adfm.201910596

31. L. Yu, I.K. Mishra, Y.L. Xie, H.Q. Zhou, J.Y. Sun et al., Ternary $\mathrm{Ni}_{2(1-\mathrm{x})} \mathrm{Mo}_{2 \mathrm{x}} \mathrm{P}$ nanowire arrays toward efficient and stable hydrogen evolution electrocatalysis under large-current-density. Nano Energy 53, 492-500 (2018). https://doi. org/10.1016/j.nanoen.2018.08.025

32. Q. Zhang, W. Chen, G.L. Chen, J. Huang, C.S. Song et al., Bi-metallic nitroxide nanodot-decorated tri-metallic sulphide nanosheets by on-electrode plasma-hydrothermal sprouting for overall water splitting. Appl. Catal. B 261, 118254 (2020). https://doi.org/10.1016/j.apcatb.2019.11825 4

33. G.X. Wang, W. Chen, G.L. Chen, J. Huang, C.S. Song et al., Trimetallic Mo-Ni-Co selenides nanorod electrocatalysts for highly-efficient and ultra-stable hydrogen evolution. Nano Energy 71, 104637 (2020). https://doi.org/10.1016/j.nanoe n.2020.104637

34. D.L. Chen, Z.M. Xu, W. Chen, G.L. Chen, J. Huang et al., Mulberry-inspired nickel-niobium phosphide on plasmadefect-engineered carbon support for high-performance hydrogen evolution. Small 16, 2004843 (2020). https://doi. org/10.1002/smll.202004843

35. D.L. Chen, Z.M. Xu, W. Chen, G.L. Chen, J. Huang et al., Just add water to split water: ultrahigh-performance bifunctional electrocatalysts fabricated using eco-friendly heterointerfacing of NiCo diselenides. J. Mater. Chem. A 8, 12035-12044 (2020). https://doi.org/10.1039/D0TA02121K

36. C. Yu, J.J. Lu, L. Luo, F. Xu, P.K. Shen et al., Bifunctional catalysts for overall water splitting: CoNi oxyhydroxide nanosheets electrodeposited on titanium sheets. Electrochim. Acta 301, 449-457 (2019). https://doi.org/10.1016/j.elect acta.2019.01.149

37. S.W. Wen, T. Yang, N.Q. Zhao, L.Y. Ma, E.Z. Liu, Ni-Co-MoO nanosheets decorated with $\mathrm{NiCo}$ nanoparticles as advanced electrocatalysts for highly efficient hydrogen evolution. Appl. Catal. B 258, 117953 (2019). https://doi.org/10.1016/j.apcat b.2019.117953

38. G.C. Yang, Y.Q. Jiao, H.J. Yan, Y. Xie, A.P. Wu et al., Interfacial engineering of $\mathrm{MoO}_{2}-\mathrm{FeP}$ heterojunction for highly efficient hydrogen evolution coupled with biomass electrooxidation. Adv. Mater. 32, 2000455 (2020). https://doi.org/10.1002/ adma.202000455

39. H.J. Yan, Y. Xie, A.P. Wu, Z.C. Cai, L. Wang et al., Anionmodulated HER and OER activities of 3D Ni-V-based interstitial compound heterojunctions for high-efficiency and stable overall water splitting. Adv. Mater. 31, 1901174 (2019). https ://doi.org/10.1002/adma.201901174

40. M. Ming, Y.L. Ma, Y. Zhang, L.B. Huang, L. Zhao et al., 3D nanoporous $\mathrm{Ni} / \mathrm{V}_{2} \mathrm{O}_{3}$ hybrid nanoplate assemblies for highly efficient electrochemical hydrogen evolution. J. Mater. Chem.
A 6, 21452-21457 (2018). https://doi.org/10.1039/C8TA0 $7701 \mathrm{~K}$

41. G.F. Qian, G.T. Yu, J.J. Lu, L. Luo, T. Wang et al., Ultrathin N-doped-graphene encapsulated $\mathrm{Ni}$ nanoparticles coupled with $\mathrm{MoO}_{2}$ nanosheets for highly efficient water splitting at large current density. J. Mater. Chem. A 8, 14545-14554 (2020). https://doi.org/10.1039/d0ta04388e

42. S.Y. Jing, L.S. Zhang, L. Luo, J.J. Lu, S.B. Yin et al., N-doped porous molybdenum carbide nanobelts as efficient catalysts for hydrogen evolution reaction. Appl. Catal. B 224, 533-540 (2018). https://doi.org/10.1016/j.apcatb.2017.10.025

43. J. Zhang, T. Wang, P. Liu, Z.Q. Liao, S.H. Liu et al., Efficient hydrogen production on $\mathrm{MoNi}_{4}$ electrocatalysts with fast water dissociation kinetics. Nat. Commun. 8, 15437 (2017). https:// doi.org/10.1038/ncomms 15437

44. M.J. Zang, N. Xu, G.X. Cao, Z.J. Chen, J. Cui et al., Cobalt molybdenum oxide derived high-performance electrocatalyst for the hydrogen evolution reaction. ACS Catal. 8, 5062-5069 (2018). https://doi.org/10.1021/acscatal.8b00949

45. D.H. Deng, K.S. Novoselov, Q. Fu, N.F. Zheng, Z.Q. Tian et al., Catalysis with two-dimensional materials and their heterostructures. Nat. Nanotechnol. 11, 218-230 (2016). https:// doi.org/10.1038/nnano.2015.340

46. X.R. Gao, X.M. Liu, W.J. Zang, H.L. Dong, Y.J. Pang et al., Synergizing in-grown $\mathrm{Ni}_{3} \mathrm{~N} / \mathrm{Ni}$ heterostructured core and ultrathin $\mathrm{Ni}_{3} \mathrm{~N}$ surface shell enables self-adaptive surface reconfiguration and efficient oxygen evolution reaction. Nano Energy (2020). https://doi.org/10.1016/j.nanoen.2020.105355

47. J. Jian, L. Yuan, H. Qi, X.J. Sun, L. Zhang et al., $\mathrm{Sn}-\mathrm{Ni}_{3} \mathrm{~S}_{2}$ ultrathin nanosheets as efficient bifunctional water-splitting catalysts with a large current density and low overpotential. ACS Appl. Mater. Interfaces 10, 40568-40576 (2018). https ://doi.org/10.1021/acsami.8b14603

48. Y.T. Luo, L. Tang, U. Khan, Q.M. Yu, H.M. Cheng et al., Morphology and surface chemistry engineering toward $\mathrm{pH}$ universal catalysts for hydrogen evolution at high current density. Nat. Commun. 10, 269 (2019). https://doi.org/10.1038/ s41467-018-07792-9

49. Y. Li, F.M. Li, X.Y. Meng, S.N. Li, J.H. Zeng et al., Ultrathin $\mathrm{Co}_{3} \mathrm{O}_{4}$ nanomeshes for the oxygen evolution reaction. ACS Catal. 8, 1913-1920 (2018). https://doi.org/10.1021/acsca tal.7b03949

50. L. Hui, Y.R. Xue, D.Z. Jia, H.D. Yu, C. Zhang et al., Multifunctional single-crystallized carbonate hydroxides as highly efficient electrocatalyst for full water splitting. Adv. Energy Mater. 8, 1800175 (2018). https://doi.org/10.1002/aenm.20180 0175

51. F. Yu, H.Q. Zhou, Y.F. Huang, J.Y. Sun, F. Qin et al., Highperformance bifunctional porous non-noble metal phosphide catalyst for overall water splitting. Nat. Commun. 9, 2551 (2018). https://doi.org/10.1038/s41467-018-04746-Z

52. X. Yu, Z.Y. Yu, X.L. Zhang, Y.R. Zheng, Y. Duan et al., "Superaerophobic" nickel phosphide nanoarray catalyst for efficient hydrogen evolution at ultrahigh current densities. J. Am. Chem. Soc. 141, 7537-7543 (2019). https://doi. org/10.1021/jacs.9b02527 
53. T. Tang, W.J. Jiang, S. Niu, N. Liu, H. Luo et al., Electronic and morphological dual modulation of cobalt carbonate hydroxides by Mn doping toward highly efficient and stable bifunctional electrocatalysts for overall water splitting. J. Am. Chem. Soc. 139, 8320-8328 (2017). https://doi.org/10.1021/ jacs.7b03507

54. X. Zou, Y.P. Liu, G.D. Li, Y.Y. Wu, D.P. Liu et al., Ultrafast formation of amorphous bimetallic hydroxide films on 3D conductive sulfide nanoarrays for large-current-density oxygen evolution electrocatalysis. Adv. Mater. 29, 1700404 (2017). https://doi.org/10.1002/adma.201700404

55. L.M. Cao, Y.W. Hu, S.F. Tang, A. Iljin, J.W. Wang et al., FeCoP electrocatalyst derived from a bimetallic prussian blue analogue for large-current-density oxygen evolution and overall water splitting. Adv. Sci. 5, 1800949 (2018). https://doi. org/10.1002/advs.201800949

56. N. Zhang, Y. Gao, Y.H. Mei, J. Liu, W.Y. Song et al., CuS$\mathrm{Ni}_{3} \mathrm{~S}_{2}$ grown in situ from three-dimensional porous bimetallic foam for efficient oxygen evolution. Inorg. Chem. Front. 6, 293-302 (2019). https://doi.org/10.1039/c8qi01148f

57. X.X. Zou, Y.Y. Wu, Y.P. Liu, D.P. Liu, W. Li et al., In situ generation of bifunctional, efficient Fe-based catalysts from mackinawite iron sulfide for water splitting. Chem 4, 11391152 (2018). https://doi.org/10.1016/j.chempr.2018.02.023 